Construire le voisin. Pratiques européennes

\title{
Editorial. Construire le voisin. Pratiques européennes
}

Editorial. Constructing the Neighbour. European Practices

\section{Thierry Balzacq}

\section{(2) OpenEdition \\ 1 Journals}

Édition électronique

URL : http://journals.openedition.org/conflits/2551

DOI : 10.4000/conflits.2551

ISSN : $1777-5345$

Éditeur :

CCLS - Centre d'études sur les conflits lilberté et sécurité, L'Harmattan

Édition imprimée

Date de publication : 17 septembre 2007

Pagination : 7-9

ISBN : 978-2-296-03458-7

ISSN : 1157-996X

Référence électronique

Thierry Balzacq, «Editorial. Construire le voisin. Pratiques européennes », Cultures \& Conflits [En ligne], 66 | été 2007, mis en ligne le 12 juillet 2007, consulté le 30 mars 2021. URL : http://

journals.openedition.org/conflits/2551; DOI : https://doi.org/10.4000/conflits.2551 


\section{Editorial. Construire le voisin. Pratiques européennes}

\section{Thierry BALZACQ}

Thierry Balzacq est professeur de science politique à l'université de Namur en Belgique et il enseigne la théorie des relations internationales à Sciences Po Paris. Il est chercheur associé an Centre for European Policy Studies (CEPS) de Bruxelles et au Centre d'études des crises et conflits internationaux (CECRI) de l'université catholique de Louvain. Ses travaux portent sur les approches critiques de la sécurité, la théorie des relations internationales et les effets de la démarcation géographique sur la politique extérieure de l'UE.

La politique européenne de voisinage (PEV) est un dispositif intégré, particulièrement ambitieux, de politique étrangère. Son but principal est de s'assurer que les frontières externes de l'Union européenne (UE) n'entravent les échanges commerciaux, la coopération régionale, les interactions sociales et culturelles. Les processus de la PEV combinent donc, d'un seul tenant, les trois piliers de l'Union ${ }^{1}$.

L'un des traits les plus fascinants de la PEV est le rapport, pas tout à fait déterminé, qu'elle entretient tant avec les principes de l'adhésion qu'avec les mécanismes du partenariat ${ }^{2}$. Examiner cette politique revient donc à clarifier, dès le départ, son statut. Il faut d'abord souligner que bien que le partenariat ait souvent été l'antichambre de l'intégration, ces politiques diffèrent l'une de l'autre par leurs outils, leurs mécanismes et leurs finalités. En effet, le processus de l'intégration européenne a été un formidable instrument de politique étrangère ${ }^{3}$. L'élargissement montre comment les normes, valeurs et institutions de l'UE imprègnent progressivement - à travers des étapes précises à franchir, des conditions à remplir et des attitudes à épouser - les structures politiques de

1. Voir Stetter S., EU Foreign and Interior Policies: Cross-pillar Politics and the Social Construction of Sovereignty, Londres, Routledge, 2007.

2. Voir le numéro spécial sur la politique européenne de voisinage de European Political Economy Review, $\mathrm{n}^{\circ} 7,2007$.

3 . Cette vision instrumentale ne doit pas occulter le caractère relationnel de l'intégration, laquelle n'a de sens qu'au sein de certains cadres sociaux. 
l'Etat-candidat. Par ce procédé, l'Etat admis passe de l'extérieur à l'intérieur de l'architecture communautaire. C'est sous ce rapport en outre, du point de vue performatif, que se dissipe la suspicion à son égard et qu'il cesse d'apparaître comme un membre du « eux » pour être indissociablement associé au « nous».

Le partenariat et l'intégration ont, à bien des égards, une même base idéologique : l'Union, dans ce contexte, est essentiellement une puissance civile qui ne cherche moins à imposer ses principes de conduite qu'à persuader ses voisins à adopter des normes politiques, économiques et sociales ${ }^{4}$. C'est cette configuration qui, en somme, donne à la politique extérieure de l'UE une certaine continuité ou une constance, en dépit des variations de contenu et de la diversité des régions couvertes.

Quand on examine la littérature sur la PEV, ce qui est frappant, ce n'est pas l'abondance de la moisson, mais l'éclat des impensés. On trouve, en effet, d'intéressantes réflexions sur l'Europe comme acteur international. Mais très peu d'études sont consacrées à ce qui en constitue l'assise, c'est-à-dire ses frontières 5 . On rencontre très souvent des textes stimulants sur le caractère réellement communautaire de la $\mathrm{PEV}$; mais l'explication généalogique et l'analyse des logiques bureaucratiques de cette construction restent peu explorées, sinon inexistantes. Enfin, il n'est pas rare de trouver, sur la dimension externe de justice et affaires intérieures, de solides travaux conceptuels. Mais l'examen demeure en général au niveau des aspérités des thèmes étudiés (par exemple : immigration et asile).

Ce numéro spécial tente d'éviter ces pièges, en se concentrant sur un aspect de la PEV, celui qui nous semble le plus structurant, en l'espèce : la sécurité. Son ambition principale est en effet de montrer que la perspective sécuritaire domine le projet et la dynamique de la PEV. Cela ne veut pas dire, pour éviter toute méprise, que les aspects économiques, sociaux, culturels ou environnementaux n'y ont pas de place. Loin s'en faut; cette place est d'ailleurs, en plusieurs endroits, notable. En revanche, et c'est là le point de convergence des contributions réunies ici, il nous semble que dans sa conception, son contenu et son déploiement, la sécurité subsume, de manière décisive, les autres volets de coopération. Il faut noter par ailleurs que les textes s'articulent autour de la sécurité intérieure et, subsidiairement, des effets qu'elle a sur la politique extérieure de l'Union. Enfin, ce numéro se concentre sur la PEV, vue de l'UE. Il nous parait adéquat d'insister sur la nécessité d'encourager d'autres chercheurs à produire des travaux portant

4. Wallace W., «Looking after the neighbourhood. Responsibilities for the EU-25 », Notre Europe, Policy Paper $\mathrm{n}^{\circ}$ 4, 2003.

5. Voir Anderson M., «La frontière : un débat contemporain », Cultures $\mathcal{E}$ Conflits, n ${ }^{\circ} 26-27$, 1997, pp. 15-34 ; Foucher M., L'Invention des frontières, Paris, Fondation des études de défense nationale, 1986. 
sur la PEV, telle qu'elle est perçue et vécue par les voisins. Cela nous permettra d'en avoir un tableau plus équilibré, et probablement, plus contrasté.

La succession des articles de ce numéro de Cultures $\mathcal{E}$ Conflits obéit à un «agencement gigogne». Le texte de Julien Jeandesboz examine les mécanismes cognitifs et les luttes bureaucratiques qui ont influencé la formation de la PEV. Il permet ainsi de faire ressortir que la sécurité est le principal thème autour duquel s'organisent les relations entre l'UE et ses voisins. Thierry Balzacq explore la problématique de la démarcation géographique et ses effets sur la sécurité régionale. Deux des idées qu'il articule pour saisir la notion de "complexe de sécurité » - la frontiérisation et la gouvernance externe - sont amplifiées, sous des angles différents, par Ruben Zaiotti et Sarah Wolff ${ }^{6}$. En partant d'une approche philosophique, le premier développe l'argument aux termes duquel le souci sécuritaire qui anime la politique de l'UE aux frontières externes, aboutit à l'institutionnalisation d'une « communauté de sécurité autiste ». Sarah Wolff analyse, quant à elle, un modèle particulier de gestion des frontières, c'est-à-dire celui offert par la coopération policière renforcée.

C'est avec enthousiasme, et en espérant que la PEV suscitera le débat qu'elle mérite, que nous offrons au lecteur les réflexions qui suivent.

6. Friis L., Murphy A. «The European Union and Central and Eastern Europe: governance and boundaries ", Journal of Common Market Studies, vol. 37, n², 1999, pp. 211-232 ; Lavenex S., "EU external governance in "Wider Europe" ", Journal of European Public Policy, vol. 11, $\mathrm{n}^{\circ} 4,2004$, pp. 680-700. 